\title{
Topographical Anatomical Neuropathic-Pain Guided (TANG) Mapping: A Tool Derived from a Patient Perspective to Facilitate the Transition from Spinal Cord Stimulator Trial to Potential Permanent Implantation
}

\author{
Nelson Tang, Bryt A. Christensen, Kayode A. Williams
}

Department of Anesthesiology \& Critical Care Medicine, Division of Pain Medicine, Johns Hopkins Hospital, Baltimore, USA. Email: kwilli64@jhmi.edu

Received April $8^{\text {th }}$, 2012; revised May 10 ${ }^{\text {th }}, 2012$; accepted June $1^{\text {st }}, 2012$

\begin{abstract}
Introduction: Collecting a patient's pain scores and the analgesic effect achieved during spinal cord stimulation (SCS) trials can be difficult, and no standard exists for doing so. We propose a topographical mapping tool that was derived from a patient's perspective. Case: A 60-year-old man with postherpetic neuralgia (PHN) underwent a SCS trial after conservative treatment failed to relieve his pain. During the SCS trial, with the SCS off and on in five different settings, he recorded pain levels in each of the six different painful zones he identified. The data collected were transferred to a topographical and anatomical map, which helped the physicians to better understand the effects of the SCS at different settings. Ultimately, the data collected by the patient helped the physicians to implant a permanent SCS successfully. Conclusions: Patient pain diaries have been used in pain medicine for years. This particular patient's collection of pain scores and SCS effects inspired the construction of a more standardized tool for collecting such data during SCS trials. We propose that use of our Topographical Anatomical Neuropathic-pain Guided (TANG) mapping tool will enable physicians to choose SCS lead positions more precisely than is currently possible.
\end{abstract}

Keywords: Neuromodulation; Spinal Cord Stimulation; Pain Mapping Tool; TANG Mapping

\section{Introduction}

In rare instances, highly descriptive, well demarcated, and precisely detailed feedback from a patient can greatly facilitate the ability of healthcare practitioners to understand the complexity of neuropathic patterns and the potential analgesic benefit from neuromodulation. In one such instance, a patient with postherpetic neuralgia (PHN) inspired us to change our approach to dealing with neuropathic pain and spinal cord stimulation (SCS), a form of neuromodulation. Although PHN is dermatomal in nature [1], some patients with PHN can have multiple different pains, dysesthesias, and hyperalgesia simultaneously, each with its own specific nature and sub regions that may extend beyond the boundaries of the original herpes zoster rash regions [2]. Such chronic pain patterns can have detrimental effects on social and psychological functioning and adversely affect quality of life [3].

PHN is one of multiple neuropathic pain conditions for which SCS may potentially provide substantial analgesic benefit and quality of life enhancement. However, the approach taken during the transition from SCS trial to permanent implantation varies widely between medical practitioners. Although some practitioners will simply ask the patient whether or not he or she experienced overall improvement during the SCS trial week, we believe that it is pivotal to seek more detailed and precise information. The approach of ascertaining maximal information regarding the patient's specific experiences during the trial week is invaluable. Such knowledge makes future implantation and reprogramming sessions much more likely to capture optimal and effective SCS coverage where it counts the most-namely what the patient feels is most analgesic and beneficial to his or her quality of life.

\section{Case Report}

A 60-year-old man with PHN presented with unremitting, chronic left thoracoscapular, axillary, and chest pain. He had been diagnosed 2 years prior with acute herpes zoster after presenting to the emergency department with a painful rash. At that time, he was prescribed acyclovir, but after the resolution of the rash, he developed constant 
pain in the above-mentioned areas. He rated his maximum pain, which was located at and adjacent to the site of his prior rash, at 7 out of 10 on the numerical pain rating scale. His pain occurred two to three times per day in episodes that lasted approximately 1 hour. He had a sharp stabbing pain in the left anterior chest that he described as feeling like he had a "hook" underneath his skin that was "suspending his weight". It was exacerbated with activities such as showering or by light touch such as from clothes rubbing against his skin. His allodynia had multiple triggers. His highly complex pain patterns included certain pain zones that each possessed its own unique characteristics, including stabbing, cramping, acidic, burning, deep, and spasmodic pain; all had an allodynic component.

The patient's pain was refractory to varying regimens of topirimate, pregabalin, Duloxetine, and oxycodone. Furthermore, interventions such as left T6 thoracic transforaminal epidural steroid injections had previously failed to bring relief. Thus, neuromodulation therapy was implemented. Neuromodulation has been described in the literature as a last resort option for PHN pain, especially after analgesic medication dosage has already been stabilized [4,5]. However, SCS has promising potential, as $82 \%$ of PHN patients in one study reported long-term relief 29 months after receiving SCS implantation [6].

The patient underwent an SCS trial that utilized an octad lead with a ligamentum flavum penetration entry point at the T12-L1 interspace. Advanced under fluoroscopy, the lead was positioned at the lower endplate of T6. Stimulation coverage of the left thoracoscapular area was confirmed.

During the week of the trial, the patient was able to provide topographical and anatomical feedback of unusually high fidelity. This feedback was a valuable and powerful tool at the time of SCS implantation, because we were far better equipped than we ordinarily are with knowledge of pain zones and coverage zones.

The patient had broad painful regions and distinctly different smaller sub regions within the broad regions. These regions differed in the nature of pain, in numerical intensity, and in descriptive nature such as acidic, burning, stabbing, etc. The patient displayed SCS trial coverage zones topographically based on varying stimulation settings. Thus, we were able to obtain the area and the location of coverage zones. The patient also included information on whether a certain SCS setting aggravated or alleviated pain from baseline or had no effect. We were able to use this information to make adjustments during permanent lead implantation to better optimize these therapeutic coverage zones to coincide with pain zones.

\section{Discussion}

Pain diaries and dermatomal mapping have been used in pain management for many years [7]. In this case, we had a pain diary in conjunction with distinct delineation of pain zone borders based on specific and differing natures of pain, as well as SCS on-off status. To the authors' best knowledge, such comprehensive feedback schematics have not been described in the medical literature in relation to SCS neuromodulation for PHN. We believe that use of such schemes should be encouraged with the multitude of neuropathic pain conditions for which SCS may prove beneficial.

During SCS trials, an awake patient may provide verbal feedback regarding SCS stimulation while lying prone on the operating room table [8]. However, this feedback can differ substantially from that given when the same patient performs activities of daily living (ADLs) during the days after the trial lead insertion [9, 10]. The pain perceptions, as well as coverage perceptions, can vary based on the activity and position of the patient. Such information cannot be overlooked when considering permanent SCS implantation.

PHN symptomatology can be complex, and the pain can be patchy and not contiguous. Having acknowledged this, there is no clear consensus about the mechanism of action for SCS neuromodulation. To date, the evidence for patient provided mapping of pain and stimulation coverage crossover is nonexistent. Most SCS implantation manuals provide a generalized guide for areas of body mapping, however; there is wide inter-individual variability with regards to mapping.

Although SCS coverage in the form of patient-reported, stimulation-induced paresthesias has been described in the medical literature [11], prior data collection attempts have not focused simultaneously on the nature and intensity of pain, SCS on-off status, and how well different SCS settings crossed over with different pain zones. Although as many as 3800 different SCS unipolar and bipolar arrays have been evaluated in the past, improvements must still be made in our ability to:

1) Determine the actual severity, precise location, boundaries, and nature of various pain zones.

2) Determine whether different stimulation settings of stimulation induced paresthesias correspond to and encompass the entirety of the pain zones or provide only partial coverage.

3) Determine qualitatively and quantitatively the analgesic benefit that different stimulation settings provide.

4) Understand that certain SCS settings that induce paresthesias can actually be more uncomfortable than the baseline pain.

A substantial amount of work has been done to ad- 
vance our understanding of the topographical mapping of the dorsal column as it relates to SCS. Such work has been carried out by computer-generated models as well as in animal and human studies [12,13]. The medical literature offers only general guidelines that establish a relationship between stimulation coverage patterns and spine levels of implanted SCS electrodes [11]. However, inter-individual variability and unique pain patterns pose complex roadblocks to achieving maximal potential analgesic benefit via SCS.

In our experience, the manifestation of pain from the patient's perspective does not always correlate with the topographical representations of the dermatomes in the dorsal column. Therefore, we have devised and now propose the following streamlined tool to help bridge this gap. Although inspired by the case presented here of the patient with PHN, the tool has been configured to be less verbose and easier to use and analyze. "Topographical Anatomical Neuropathic-pain Guided” (TANG) mapping (Figure 1) will rely on highly specific patient feedback. However, we have formatted this tool to emphasize ease of understanding and use while maximizing precise gathering of information during the trial period; this information is expected to be highly individualized for each patient. As seen in the comprehensive mapping system, a practitioner can intuitively and easily extrapolate pain zone borders, pain zone intensity, and pain zone nature with the patient at rest and during ADLs and with the SCS on and off. From these data, the practitioner can predict various SCS setting coverage boundaries and, most importantly, the analgesic effectiveness at different SCS settings.

To use the TANG mapping tool, the patient is asked to pick differently sized alphabetic letters that correspond to the nature(s) of his or her pain. Often, different types of pain will coexist simultaneously. For example, the letter $\mathrm{B}$ denotes burning, and the letter $\mathrm{S}$ denotes stabbing. Larger fonts represent higher numerical pain scores. As illustrated in our example TANG mapping tool (Figure 1), the patient marks the area of his or her baseline pain with the appropriate letter on an anatomical figure diagram that is initially an empty canvas. The first canvas is used to denote the pain patterns when the SCS is off. The patient may mark the same letter numerous times if a certain pain zone encompasses a broad region. The information localizes and differentiates the baseline pain zones in a very specific fashion and demarcates neighboring sub regions and certain hot zones where the pain is worst. We have discovered that such hot zones can be embedded within larger and broader pain zones and yet be of a distinctly different nature and quality from the broader pain zone. The process is then repeated with the SCS turned on at different settings to delineate bounda-

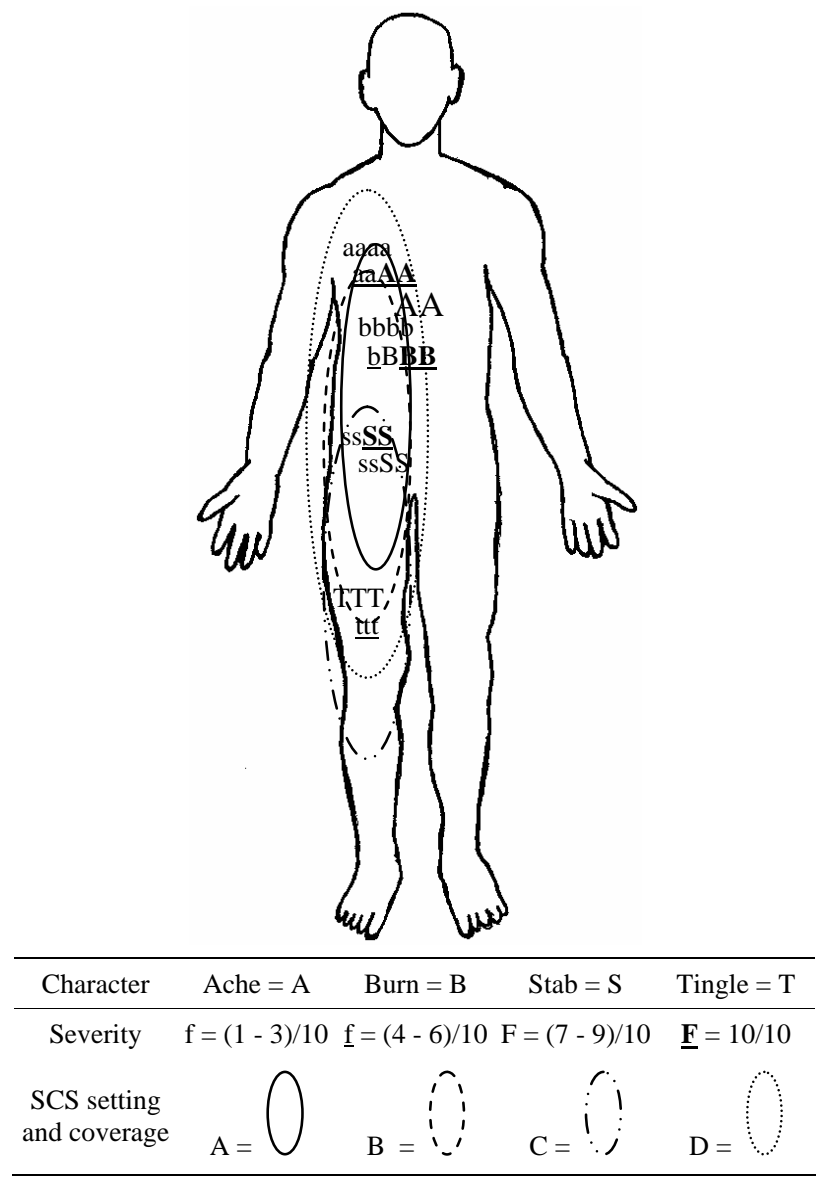

Figure 1. Example of how the front side of the TANG Mapping Tool might look after a patient has filled out the pain zones and spinal cord stimulation (SCS) coverage zones during an SCS trial period. The legend is shown below the figure.

ries of different SCS coverage zones.

By comparing the pain patterns made with SCS on and those made with SCS off, we can gain an understanding of which SCS settings cover the largest area. In other words, we can determine whether any SCS setting covers all of the pain zones in their entirety. We can also determine which SCS settings are most effective at reducing the level of baseline pain. The amount by which alphabetic character font size is reduced between the SCS-off and SCS-on figures provides a much clearer picture about multiple painful sub-zones than does asking the patient how much pain improved when the SCS was turned on, with or without an accompanying pain diary that uses a numeric rating scale or visual analog scale.

Even more useful is having the patient use the tool to map his pain with the SCS on or off during ADLs. This information is critical because in many cases, SCS provides analgesic benefit when the patient is at rest but does not adequately control pain when the patient carries 
out certain movements or activities that are inherent to conducting ADLs. Initially derived from a patient perspective (Figure 2), the TANG mapping tool can potentially be used to improve quality of life and meet ADL expectations, during the trial period. Thus, as seen in the case presented here, a pain-mapping tool can help to prioritize and facilitate precise targeting for subsequent SCS analgesic coverage when the time comes for permanent SCS implantation.

\section{Conclusions}

As illustrated in the patient's case described above, use of a tool such as a patient-orchestrated mapping system empowers the physician with knowledge about what the patient is specifically experiencing during the SCS trial week. The information acquired is far more objective and detailed than that obtained from subjective responses to questions regarding whether patient's pain was better, the same, or worse during the trial week. The very focal and precise information gained during the trial period allows a physician to implant the stimulator permanently with more confidence that the intricacies of pain zones and pain sub-zones are well understood.

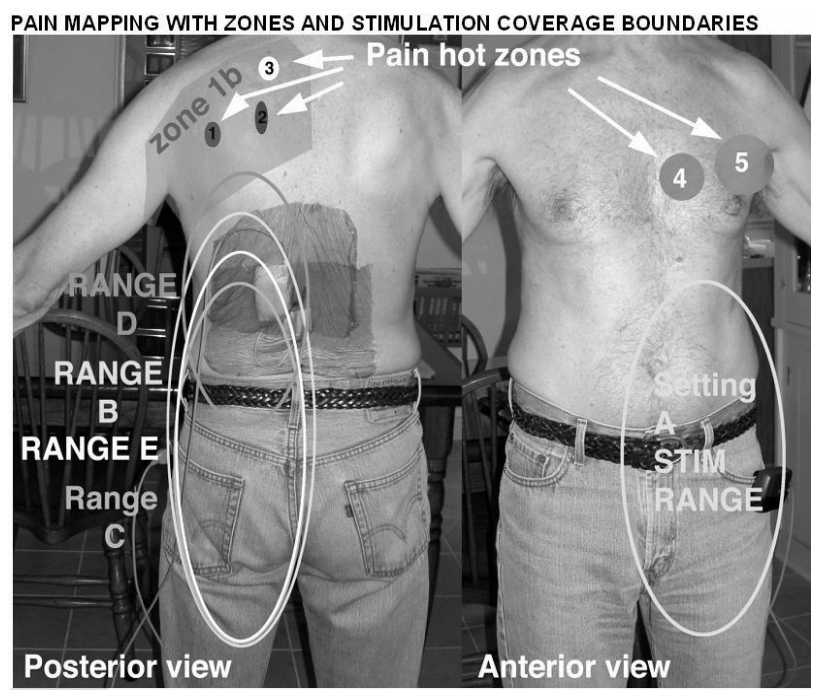

Pain Zones:
Zone 1: Most frequent area of local, dagger-like, intense pain, typically level 8 , contact-induced, although sometimes spasms without any obvious trigger.

Zone 1b: Broader area of constant, severe sunburn pain, disrupts daily activity like wearing clothes Zone 2: Second-most frequent area of local, dagger-like, intense pain, typically level 8 , contact-

Zone 3: Deep, intense, knot-like ache, typically level 6-7, always present, with varying intensity

Zone 4: Third-most frequent area of local, dagger-like, intense pain, typically level 7, contact-induced; Zone 5: Fourth-most frequent ain in this zone

(iker-like, intense pain, typically level 9 , most severe but drom a hook; additionally, constant sunburn pain in this zone.

Stimulation settings $A, B, C, D$, and $E$ with ranges of coverage.

When stimulator settings are high, sensation occurs over the following ranges:

A-- Anterior, upper left thigh to 8 inches above waist, left abdomen area. Below all pain areas.

B-- Posterior only. 10 inches above waist/mid-back down to upper thigh

C-- Posterior only, feels lower than Zones $1-3$, from 6 inches above waist down to mid thigh. D-- Posterior only, slightly below waist up to mict-back, 4 inches below Zones $1-2$. Furthest posterior.
E-- Posterior only, 8 inches above waist extending down to left thigh.

Figure 2. Original mapping as presented from the patient's perspective.
It is our position that decisions regarding permanent lead placement (e.g. lead location), should not be based solely on generalized dorsal column topographical representations. On the contrary, such technical decisions should be based on exquisite patient mapping feedback that is collected preemptively during the trial period. This approach may enable the physician to achieve more effective SCS coverage for the patient when he or she is transitioned from SCS trial to permanent implantation. A study is ongoing to validate the mapping tool proposed in this report.

\section{Acknowledgements}

The authors would like to thank Mr. Decker for granting permission to share this information presented in this manuscript, and for his gracious co-operation in the process.

\section{REFERENCES}

[1] R. H. Dworkin and R. K. Portenoy, "Pain and Its Persistence in Herpes Zoster,” Pain, Vol. 67, No. 2-3, 1996, pp. 241-251. doi:10.1016/0304-3959(96)03122-3

[2] R. H. Dworkin, J. W. Gnann Jr., A. L. Oaklander, S. N. Raja, K. E. Schmader and R. J. Whitley, "Diagnosis and Assessment of Pain Associated with Herpes Zoster and Postherpetic Neuralgia,” Journal of Pain, Vol. 9, No. S1, 2008, pp. S37-S44. doi:10.1016/j.jpain.2007.10.008

[3] A. Philip and R. Thakur, "Post Herpetic Neuralgia," Journal of Palliative Medicine, Vol. 14, No. 6, 2011, pp. 765773. doi:10.1089/jpm.2011.9685

[4] H. T. Benzon, K. Chekka, A. Darnule, B. Chung, O. Wille and K. Malik, "Evidence-Based Case Report: The Prevention and Management of Postherpetic Neuralgia With Emphasis on Interventional Procedures,” Regional Anesthesia \& Pain Medicine, Vol. 34, No. 5, 2009, pp. 514-521. doi:10.1097/AAP.0b013e3181b137bd

[5] A. J. van Wijck, M. Wallace, N. Mekhail and M. van Kleef, "Evidence-Based Interventional Pain Medicine According to Clinical Diagnoses. 17. Herpes Zoster and Post-Herpetic Neuralgia,” Pain Practice Vol. 11, No. 1, 2011, pp. 88-97. doi:10.1111/j.1533-2500.2010.00428.X

[6] H. Harke, P. Gretenkort, H. U. Ladleif, P. Koester and S. Rahman, "Spinal Cord Stimulation in Postherpetic Neuralgia and in Acute Herpes Zoster Pain,” Anesthesia \& Analgesia, Vol. 94, No. 3, 2002, pp. 694-700. doi:10.1097/00000539-200203000-00040

[7] S. A. Greenberg, "The History of Dermatome Mapping," Archives of Neurology, Vol. 60, No. 1, 2003, pp. 126-131. doi:10.1001/archneur.60.1.126

[8] S. M. Falowski, A. Celii, A. K. Sestokas, D. M. Schwartz, C. Matsumoto and A. Sharan, "Awake vs. Asleep Placement of Spinal Cord Stimulators: A Cohort Analysis of Complications Associated With Placement," Neuromodulation: Technology at the Neural Interface, Vol. 14, No. 2, 

to Facilitate the Transition from Spinal Cord Stimulator Trial to Potential Permanent Implantation

2011, pp. 130-135.

doi:10.1111/j.1525-1403.2010.00319.x

[9] T. Cameron and K. M. Alo, "Effects of Posture on Stimulation Parameters in Spinal Cord Stimulation," Neuromodulation: Technology at the Neural Interface, Vol. 1, No. 4, 1998, pp. 177-183.

doi:10.1111/j.1525-1403.1998.tb00014.x

[10] E. Ross and D. Abejon, "Improving Patient Experience with Spinal Cord Stimulation: Implications of PositionRelated Changes in Neurostimulation,” Neuromodulation: Technology at the Neural Interface, Early View Online Version. doi:10.1111/j.1525-1403.2011.00407.x

[11] G. Barolat, F. Massaro, J. He, S. Zeme and B. Ketcik,
"Mapping of Sensory Responses to Epidural Stimulation of the Intraspinal Neural Structures in Man," Journal of Neurosurgery, Vol. 78, No. 2, 1993, pp. 233-239. doi:10.3171/jns.1993.78.2.0233

[12] J. Holsheimer, "Computer Modeling of Spinal Cord Stimulation and its Contribution to Therapeutic Efficacy," Spinal Cord, Vol. 36, No. 8, 1998, pp. 531-540. doi:10.1038/sj.sc.3100717

[13] J. Holsheimer, "Which Neuronal Elements Are Activated Directly by Spinal Cord Stimulation,” Neuromodulation: Technology at the Neural Interface, Vol. 5, No. 1, 2002, pp. 25-31. doi:10.1046/j.1525-1403.2002._2005.x 